\title{
AS BACIAS PROTEROZÓICAS DO ESPINHAÇO E SÃO FRANCISCO EM MINAS GERAIS: UMA ABORDAGEM DO PONTO DE VISTA DA ESTRATIGRAFIA DE SEQUÊNCIAS
}

\author{
Rogério Rodrigues da Silva (*)
}

\begin{abstract}
This work deals with Sequence Stratigraphy concepts applied to the Proterozoic record of the São Francisco Craton in Minas Gerais, southeast Brazil. The cratonic cover is characterized by eight sequence boundaries (unconformity and/or correlative conformity), which subdivide the Proterozoic stratigraphy into seven depositional sequences. These sequences initially filled an intracratonic rift basin (the Espinhaço Basin), which evolved into a passive continental margin (the São Francisco Basin). The Espinhaço Basin was filled by six stacked depositional sequences. Their sequence boundary unconformities are indicative of taphrogenic periods, when tectonic displacements were mainly accomodated along a master listric fault, but also along synthetic and antithetic subsidiary normal faults. The unconformities are covered by coarse alluvial conglomerates, deposited by the combined effects of source area uplift and depocenter subsidence. The depositional sequences of the Espinhaço Basin show impressive cyclical depositional patterns. They usually show the unconformities and the basal conglomerates covered by shallow-marine transgressive sediments. The São Francisco Basin, a passive continental margin, was developed at approximately $0.9 \mathrm{Ga}$. Coevally, a continental glatiation occurred over the craton, which produced a relative fall in the sea level, erosion and hence unconformity development. During that time, the basin was characterized by the deposition of tillites on the proximal reachs and turbidites on the distal portions. A transgressive phase followed the glaciation and was marked by the deposition of fine-grained sediments (lutites) and carbonatic rocks overlaying the cratonic areas. Around $0.6 \mathrm{Ga}$, during the Brasiliano Orogeny, the Espinhaço and São Francisco Basins were inverted.
\end{abstract}

\section{INTRODUÇÃO}

Este trabalho sintetiza os conhecimentos adquiridos pelo autor no decorrer dos últimos 11 anos de pesquisas sobre as coberturas Proterozóicas da borda SE do Craton do São Francisco, em Minas Gerais. O registro das coberturas cratônicas da região é caracterizado por oito discordâncias. Essas discordâncias subdividem a coluna estratigráfica em sete sequências deposicionais. Partindo do mapeamento detalhado dos limites destas sequências em pontos chaves da região, de perfis sedimentológicos dos conglomerados basais, da reconstrução paleogeográfica e da proveniência dos sedimentos, o objetivo desse artigo é apresentar uma nova proposta de subdivisão estratigráfica baseada em unidades limitadas por discordâncias (Fig. 1).

$\mathrm{O}$ conceito de sequência adotado aqui corresponde ao originalmente proposto por Sloss et al. (1949), Krumbein \& Sloss (1956) e Sloss (1963). Posteriormente, esse conceito evoluiu, com o advento da sismo-estratigrafia, para as sequências deposicionais de Mitchum et al. (1977) e Vail et al. (1977). A Comissão Norte-Americana de Nomenclatura Estratigráfica (1983: 865) e o Subcomite Internacional de Classificação Estratigráfica (Salvador, 1987) reconheceram formalmente o uso de unidades estratigráficas limitadas por discordâncias.

Algumas das sequências definidas nesse artigo correspondem a unidades litoestratigráficas, ou mesmo correspondem a associações destas. Nesses casos, prefiriu-se manter os nomes originais para não confundir mais ainda o já confuso arcabouço estratigráfico. Esse mesmo procedimento foi adotado por Dominguez (1993), quando da revisão aloestratigráfica dos sedimentos das bacias Espinhaço e São Francisco no Estado da Bahia.

\section{ARCABOUÇO GEOLÓGICO REGIONAL}

A Serra do Espinhaço em Minas Gerais está localizada na margem sudeste do Craton do São Francisco. Nesta região, sobre um embasamento Transamazônico, com idade superior a 2.0-1.8 Ga, se desenvolveram duas bacias sedimentares: um rift intracratônico de idade Paleo- a Mesoproterozóica (Bacia Espinhaço), sobreposto por uma bacia do tipo margem passiva (Bacia São Francisco), de idade Neoproterozóica.

Alguns autores preconizam a existência de uma orogênese de Idade Pós-Transamazônica/PréBrasiliana, que teria afetado a região (Orogênese Espinhaço - Almeida-Abreu, 1993; Brito-Neves et al. 1995; entre outros). Os registros deste evento tectonotermal não foram confirmados e a sua existência na região ainda é muito polêmica (Uhlein et al., 1986; Uhlein, 1991; Trompette et al., 1992; Schobbenhaus, 1993). Os depósitos de cobertura e seu embasamento apresentam-se deformados e metamorfisados devido aos efeitos compressivos da orogênese Brasiliana. Portanto, considera-se que a evolução sedimentar abrangeu o intervalo entre 1.8-0.6 Ga, valores que representam as idades das orogêneses Transamazônica e Brasiliana, respectivamente (Schobbenhaus, 1993). 


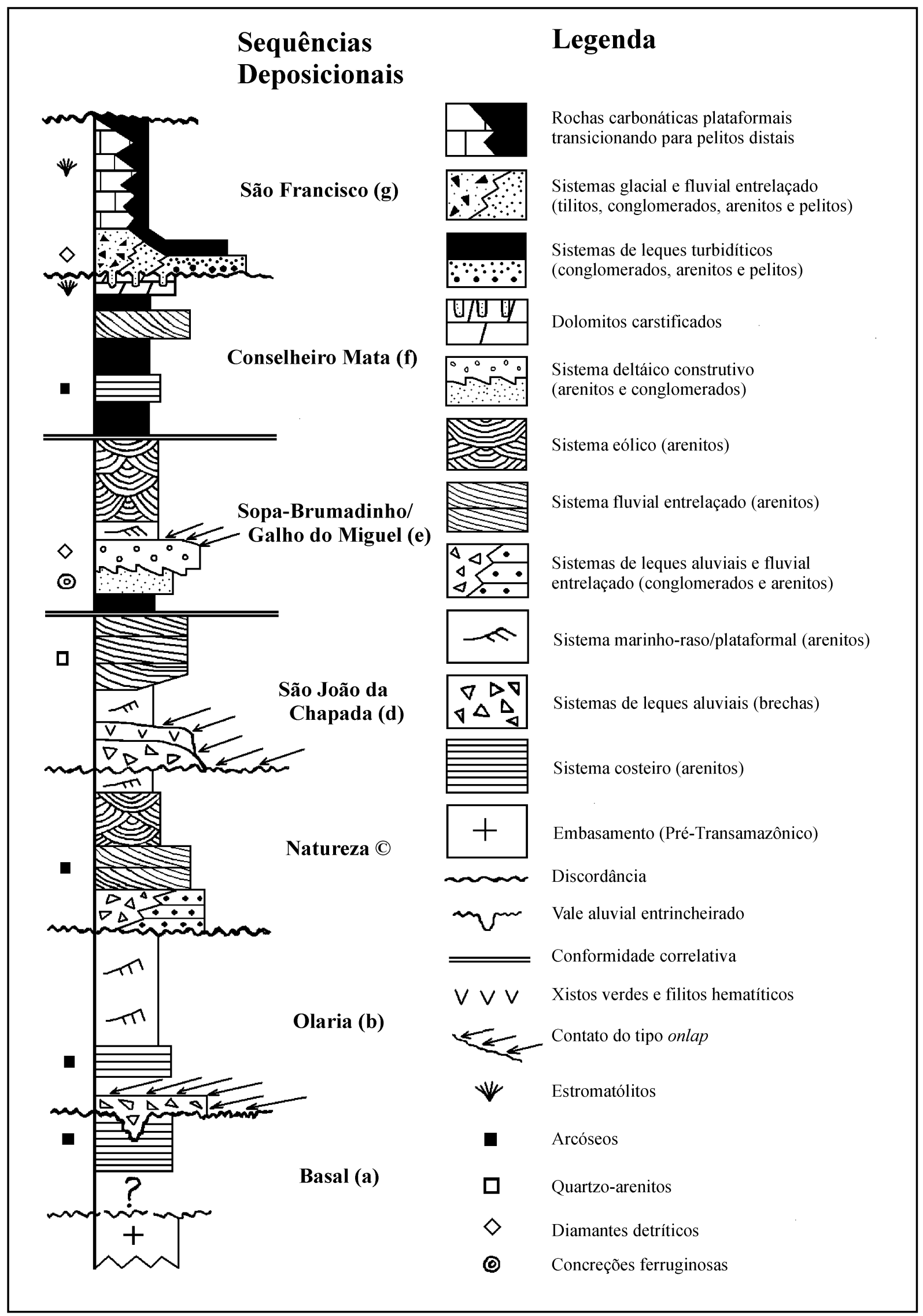

Figura 1: Coluna aloestratigráfica esquemática das coberturas Proterozóicas do Craton do São Francisco na Serra do Espinhaço, Minas Gerais. As espessuras das sequências deposicionais não foram consideradas.

Figure 1: Schemmatic allostratigraphic column of the Proterozoic cratonic covers of the São Francisco Craton in the Serra do Espinhaço, Minas Gerais. The thickness of the depositional sequences were not take into account. 


\section{Embasamento Pré-Transamazônico}

O embasamento é constituido de granitos, gnaisses, migmatitos, faixas do tipo greenstone, corpos granitóides intrusivos e corpos isolados de anfibolitos. Monzogranitos, localmente porfiríticos, predominam sobre granodioritos, tonalitos e trondjemitos (Schöll \& Fogaça, 1981). As rochas mais antigas são gnaisses migmatíticos, intrudidos por granitos peraluminosos do "tipo S" (Hoffmann, 1983; Hoppe et al., 1987). Datações em zircões indicam uma idade de 2.8 Ga para a cristalização dos granitos e uma idade de $1.8 \mathrm{Ga}$ para o seu metamorfismo, ocorrido durante a Orogênese Transamazônica (Machado et al., 1989). Corpos granitóides intrusivos de natureza calcio-alcalina ocorrem isoladamente dentro do complexo ígneomigmatítico do embasamento, na porção leste da Serra do Espinhaço em Minas Gerais. Dados geocronológicos indicam que a cristalização dessas rochas ocorreu a 1.7 Ga (Dossin, 1994).

Na porção central da cordilheira, imbricados com rochas do complexo ígneo-migmatítico, ocorrem sucessões vulcano-sedimentares, do tipo greenstonebelts (Fogaça et al., 1984). Na base dessas sucessões predominam rochas vulcânicas metamorfisadas, sobrepostas por uma unidade vulcano-sedimentar. Camadas delgadas de BIFs, chert e rochas vulcânicas, cuja composição varia de ácida a básida, ocorrem intercalados na base desta sucessão. Datações em zircões forneceram a idade de 2.9 Ga para a cristalização de riolito encontrado intercalado dentro dessa unidade (Machado et al., 1989).

\section{A Bacia Espinhaço em Minas Gerais}

Seis sequências deposicionais preencheram a Bacia Espinhaço: Basal, Olaria, Natureza, São João da Chapada, Sopa-Brumadinho/Galho do Miguel e Conselheiro Mata (Silva, 1993; 1994; 1995a; 1995b). As sequências deposicionais Basal, Olaria e Natureza afloram em uma área restrita, localizada imediatamente a SW de Diamantina. O mapeamento geológico datalhado dessa área, associado a análise paleogeográfica, permitiram caracterizar essas sequências (Silva, 1995a). As três sequências basais correspondem em conjunto à Formação Bandeirinha, definida por Fogaça et al. (1984), tido então como a porção superior da sequência greenstone de idade Arqueana, de origem epiclástica.

\section{Sequência Deposicional Basal}

Seus contatos basais são tectônicos e seu contato de topo é uma discordância com a Sequência Deposicional Olaria. Ela aflora em faixas limitadas e devido a inexistência de boas exposições não foi possível a realização da análise paleogeográfica. Os sedimentos são arcóseos, tipicamente provenientes de área fonte granítica, intracratônica.

\section{Sequência Deposicional Olaria}

A base dessa sequência é uma discordância, recoberta por corpos conglomeráticos, originados em leques aluviais e mantos de pedimento (Fig. 2).

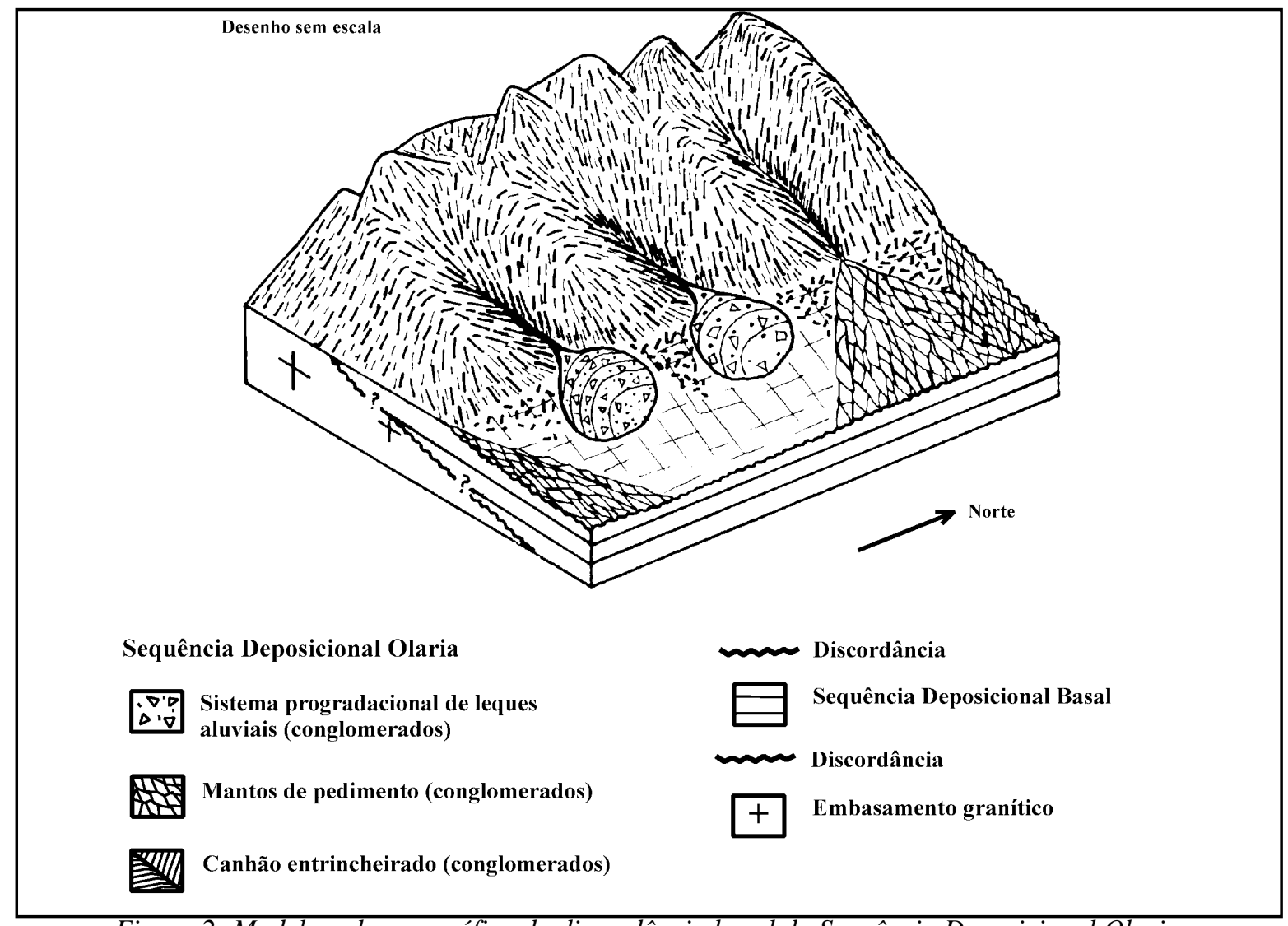

Figura 2: Modelo paleogeográfico da discordância basal da Sequência Deposicional Olaria.

Figure 2: Paleogeographic model for the basal unconformity of the Olaria Depositional Sequence. 
Os leques aluviais são constituidos por camadas métricas de conglomerados clasto-suportados. Os clastos são em sua maioria blocos e matacões. Dentro dos canhões entrincheirados, os conglomerados são sustentados pela matriz e compostos por clastos de todos os tamanhos. Clastos angulosos perfazem mais de $90 \%$ da fração grosseira. Esses apresentam seus eixos maiores orientados preferencialmente na posição vertical. Os clastos são basicamente compostos de arenitos, rochas ígneas plutônicas metamorfisadas e BIFs. A matriz dos conglomerados possui a composição de arenitos arcoseanos, entretanto abundam grandes quantidades de óxidos de ferro detríticos. A deposição dos conglomerados ocorreu a partir de fluxos de detritos relacionados a tectonismo sin-sedimentar. O soerguimento tectônico nas regiões de cabeceira causou a incisão dos vales aluviais e a consequente deposição de leques progradacionais. Dados de paleocorrentes indicam que a dispersão de sedimentos ocorreu de oeste para leste.

O pedimento estudado é uma superfície planar, porém irregular com inúmeros sinais de escavamento. O seu manto ocorre como uma camada de conglomerado matrizsuportada, localmente com até $50 \%$ de matriz arenoargilosa. Possui espessura constante e regular de 1,6 m e apresenta estratificação plano-paralela mal desenvolvida. É composto, em sua maioria, por blocos e seixos arredondados a angulosos. Os clastos são constituidos de arenitos, quartzo de veios, BIFs e granitos. $\mathrm{O}$ transporte de sedimentos ocorreu por processos trativos, principalmente por rolamento em um fluxo de correntes de alta energia. Blocos de arenitos angulosos representam o produto da erosão local de sedimentos previamente litificados. As paleocorrentes indicam transporte de detritos de W para E.

O conglomerado basal é recoberto por uma sucessão de espessura decamétrica de arenitos transgressivos de origem marinho-raso e costeiro. Os contatos basais desses arenitos são do tipo onlap. A presença de tidal bundles limitados por superfícies de reativação são indicativos fortes de deposição por correntes de marés entremeados por exposição subaérea dos sedimentos durante os períodos de retração das correntes. O padrão de paleocorrentes desses depósitos é bipolar, para NW e SE. Padrões de interferência de marcas de onda com marcas de corrente também foram observados. $\mathrm{O}$ conjunto de dados sugere que correntes transgressivas direcionadas para NW, ao longo de uma linha de costa de direção NWSE, interferiu com ondas, com direção de propagação perpendicular (Silva, 1995a).

\section{Sequência Deposicional Natureza}

O contato basal dessa sequência é uma discordância, recoberta por conglomerados heterogêneos, lateralmente persistentes, representativo de um arranjo paleogeográfico complexo de natureza aluvial (Fig. 3). Nas porções proximais da bacia, a discordância é angular porque falhamentos normais causaram o basculamento dos estratos da sequência sotoposta. A deposição ao longo dos falhamentos é caracterizada predominantemente por conglomerados oriundos da litificação de fluxos de detritos não-coesivos. Para leste, os estratos acima e abaixo da discordância são concordantes, entretanto foram observados evidências de erosão e exposição subaéreas. Nessas porções, a discordância erosiva é recoberta por um sistema de leques aluviais. Esse sistema prograda para NE, sobre um sistema fluvial conglomerático do tipo entrelaçado. A discordância basal passa então para uma paraconformidade (Silva, 1995a). Os conglomerados fluviais são clasto-suportados com até $20 \%$ de matriz arcoseana. Apresentam-se com estratificação planoparalela bem desenvolvida. Os clastos são, principalmente, seixos grandes e blocos pequenos, bem arredondados e usualmente imbricação. Nesse sistema, o transporte de detritos ocorreu por processos trativos em um fluxo de correntes de alta energia, não canalizado e direcionado para SE.

O conglomerado basal dessa sequência é recoberto por um sistema fluvial arenoso entrelaçado. Os arenitos possuem granulometria fina e estão arranjados em ciclos caracterizados pela presença de cosets de estratificação cruzada tabular, tangencial na base dos sets, localmente recobertos por arenitos com estratificação plano-paralelo e cruzada de baixo ângulo. Os estratos cruzados tabulares apresentam tendência estrato-crescentes em direção ao topo, entretanto não são grano-crescentes. Esses ciclos registram macroformas fluviais, compostas por campos de dunas bidimensionais, que em direção ao topo dão lugar a arenitos com estratificação plano-paralelo e cruzadas de baixo ângulo, provavelmente representativos dos sedimentos de stoss-side dessas formas de leito. $\mathrm{O}$ aumento cíclico da energia sugere que pulsos tectônicos atuaram durante a deposição.

Sobreposto a esse sistema ocorrem arenitos, interpretados como o registro estratigráfico de paleocampo de dunas de origem eólica. Essa interpretação é suportada pela alta maturidade textural dos arenitos finos; pela presença de estratos cruzados de grande porte; pela existência de superfícies erosivas com alta inclinação dentro de estratos cruzados e também pela superposição de marcas balísticas do tipo transladante. Os campos de dunas são constituidos principalmente por macroformas tridimensionais, apresentando até 10 metros de espessura por 60 metros de largura. Dunas eólicas bidimensionais também foram reconhecidos.

Em um dos perfis apresentados por Silva (1995a), recobrindo os paleo-campos de dunas eólicas, ocorre uma sucessão de $14 \mathrm{~m}$ de espessura de arenitos finos a muitofinos, com marcas de corrente e de onda. As marcas de corrente são predominantes, com cristas lineares, levemente onduladas e orientadas NE-SW. A direção de transporte de detritos aponta um fluxo orientado para NW. As marcas de onda são simétricas e possuem as cristas retas, pontudas e orientadas NW-SE. Foi observado um padrão de interferência entre os dois distintos tipos de ripples. A ação de ondas com cristas orientadas NW-SE, paralelas a linha de costa, foi responsável pelo desenvolvimento das marcas de onda. Fluxos marinhos transgressivos, unidirecionais para NW, ao longo da linha de costa, foram os responsáveis pela deposição das marcas assimétricas. 


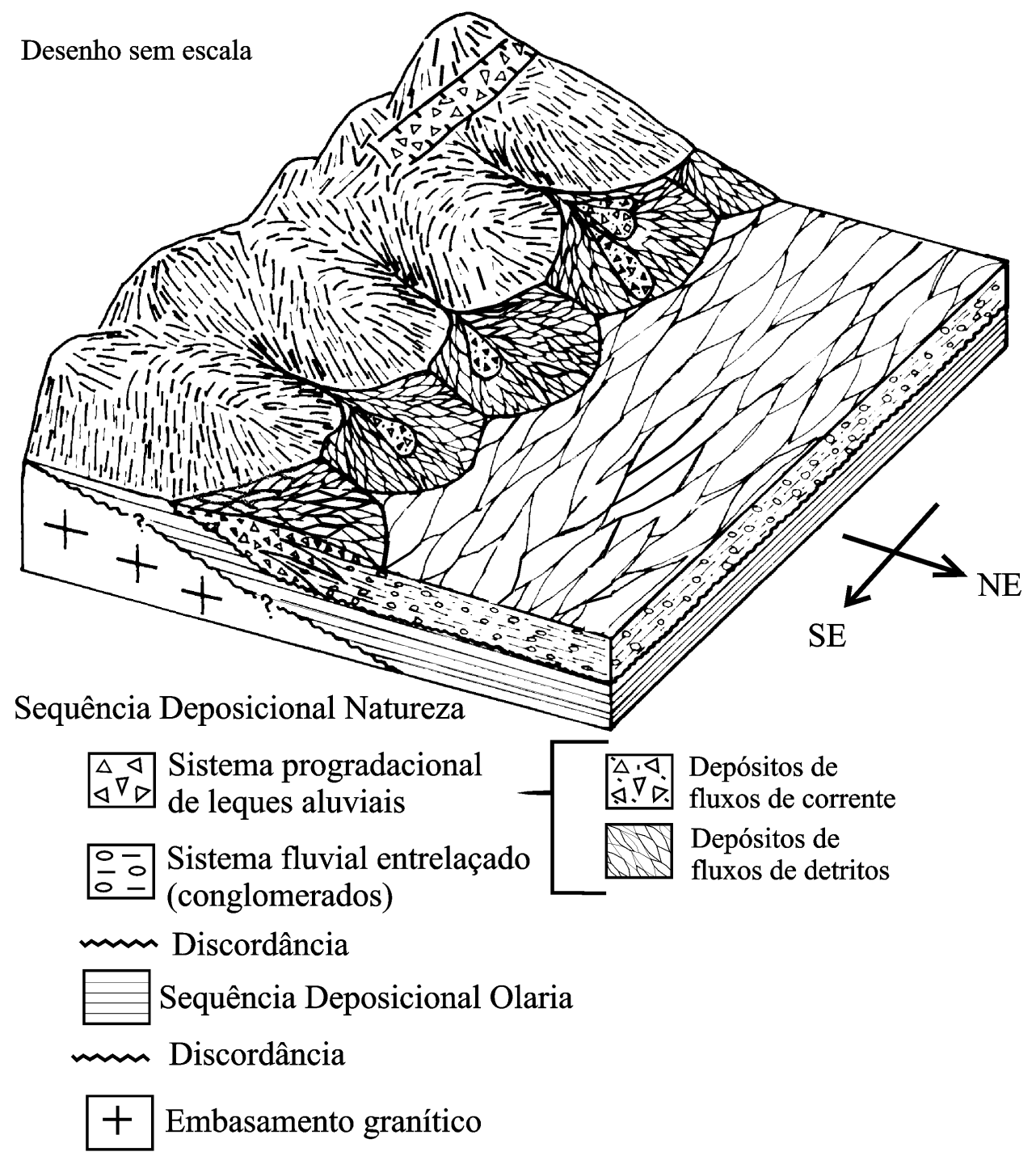

Figura 3: Modelo paleogeográfico da discordância basal da Sequência Deposicional Natureza.

Figure 3: Paleogeographic model for the basal unconformity of the Natureza Depositional Sequence.

Sequência Deposicional São João da Chapada

Esta sequência equivale a formação homônima definida por Pflug $(1965,1968)$. O seu contato basal é uma discordância angular marcante, parcialmente recoberto por brechas derivadas da litificação de depósitos de talus. Inúmeros afloramentos a $\mathrm{SW}$ de Diamantina expõem de forma espetacular essa discordância, como apresentado por Schöll \& Fogaça $(1979,1981)$, Schöll (1980), Fogaça et al. (1984), Almeida-Abreu (1989) e Silva (1993, 1994, 1995a, 1995b). Devido a esses afloramentos, Fogaça et al. (1984) postularam a existência de uma unidade litoestratigráfica pré-Espinhaço, denominada Formação Bandeirinha.

A Figura 4 apresenta um perfil geológico da discordância basal da Sequência Deposicional São João da Chapada. As brechas que recobrem a discordância são clasto-suportadas com pouca matriz. Localmente foram observados transições abruptas laterais para arenitos maciços contendo clastos angulosos. Seus clastos são predominantemente matacões pequenos e angulosos, muitos com formato tridimensional de paralelogramos (eixo $a>b>c$ ). Clastos com faces triangulares são comuns. As brechas possuem acamamento interno plano-paralelo mal desenvolvido, dado pela orientação preferencial das faces maiores dos clastos. Esses consistem basicamente de arenitos oriundos da sequência inferior, entretanto, nas porções proximais, ocorrem blocos de rochas ígneas metamorfisadas, indicando que o embasamento estava exposto a erosão subaérea.

O desenvolvimento da estratificação plano-paralela na brecha está relacionado a existência de pulsos tectônicos, provavelmente representativos de deslizamentos sucessivos ao longo das falhas sintéticas. Durante cada pulso tectônico foi depositado uma camada de brecha. A inclinação original da rampa 
Sequência Deposicional

São João da Chapada
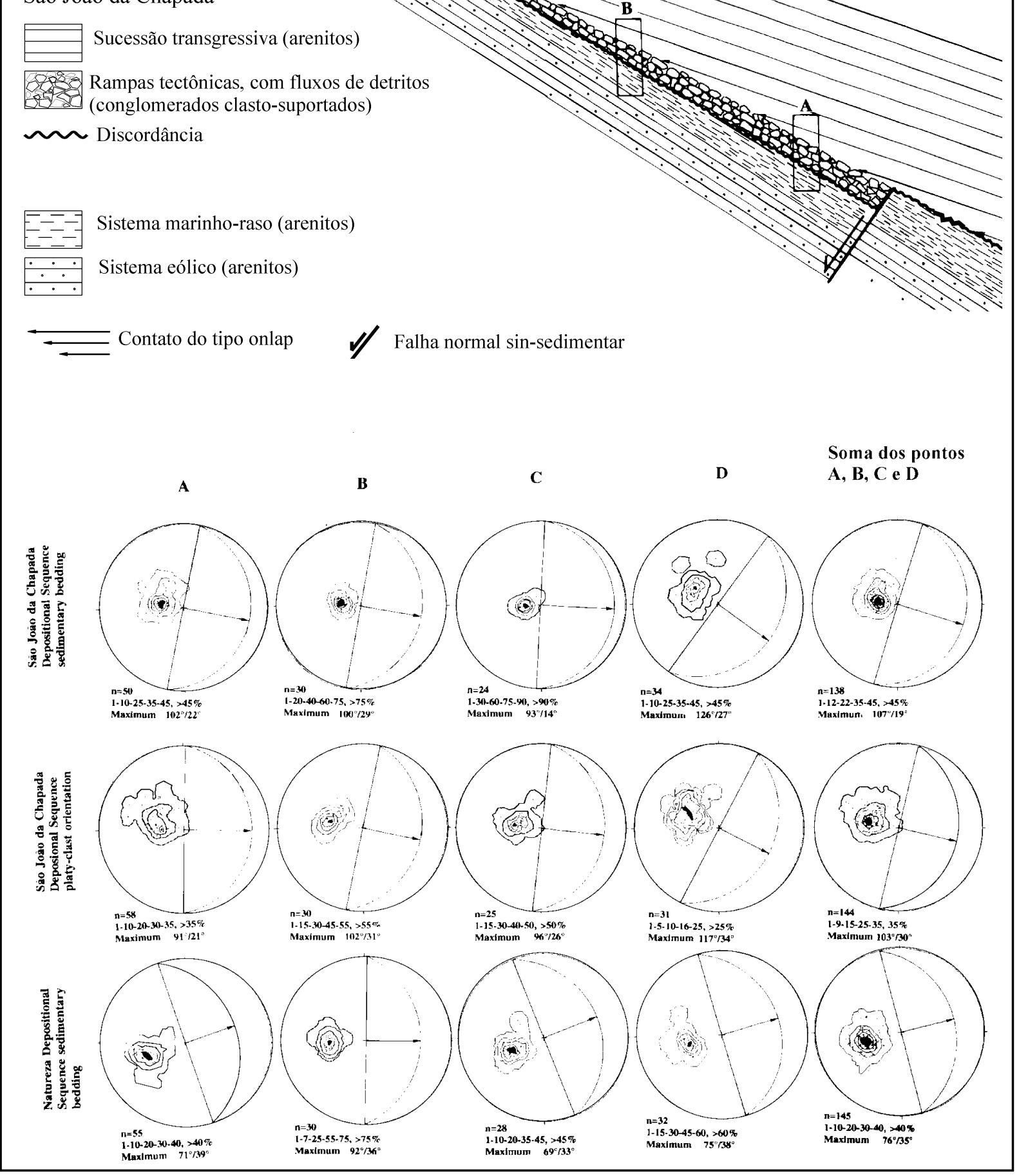

Figura 4: Perfil geológico da discordância basal da Sequência Deposicional São João da Chapada. Figure 4: Geologic cross-section of the basal unconformity of the São João da 
apresentada na Figura 4 é de aproximadamente $11^{\circ}$. Em síntese: a deposição das brechas basais dessa sequência está relacionada à exposição e erosão subaéreas da sequência sotoposta, falhamentos normais sintéticos, basculamento de blocos e o desenvolvimento de rampas tectônicas inclinadas.

Uma sucessão de xistos verdes e filitos recobrem a discordância e a brecha basal da Sequência Deposicional São João da Chapada. Predominam amplamente clorita filitos e menores intercalações de hematita filito, sericita filito e hematita-sericita filito. A interpretação corrente é que essa sucessão representa os produtos do metamorfismo e da deformação de protólitos de origem vulcânica básica (Renger, 1970; Hoppe, 1978). Os filitos hematíticos representam o produto de metamorfismo sobre perfis de alteração superficial sobre rochas ígneas de composição básica, em alguns locais podendo representar verdadeiras paleolateritas (Knauer \& Schrank, 1993).

Datações U/Pb em zircões obtidos nos filitos hematíticos apontam a idade de 1.7 Ga para a cristalização original do protólito vulcânico (Dossin et al., 1993; Dossin \& Dossin 1995). Essa datação coincide com a cristalização dos riolitos da região de Conceição do Mato Dentro, borda leste da Serra do Espinhaço Meridional (Brito-Neves et al., 1979; Machado et al., 1989). Essa idade deve ser entendida como uma etapa evolutiva da fase rift, marcada pela intensa atividade vulcânica de caráter bimodal, típico em rifts continentais.

Recobrindo a discordância basal da Sequência São João da Chapada, os conglomerados basais e as rochas metavulcânicas ocorre uma sucessão transgressiva, caracterizada pelo contato do tipo onlap (Silva, 1995a). Progradando para leste, por sobre os depósitos transgressivos basais, ocorreu o desenvolvimento de uma vasta planície fluvial arenosa do tipo entrelaçado, descrito em detalhe por Martins-Neto (1989, 1993, 1994). O sistema fluvial é compostos por ciclos arenosos com tendência grano- e estrato-decrescentes.

A origem do sistema fluvial arenoso da Sequência Deposicional São João da Chapada é devida a conjunção de três fatores: a) uma transgressão inicial gerou espaço de acomodação sub aérea, i.e. espaço para acomodação de sedimentos continentais; b) o embasamento estava, originalmente, saprolitizado e portanto suscetível a erosão e transporte, i.e. o embasamento estava preparado para produzir uma grande quantidade de detritos e c) devido ao clima semi-árido, com precipitações meteóricas torrenciais e runoff elevado (Martins-Neto, 1993; 1994). A hipótese de que a origem dos mesmos depósitos tenha sido controlada por tectônica sin-sedimentar é descartada devido a inexistência de padrões cíclicos grano- e/ou estrato-crescentes.

Sequência Deposicional Sopa-Brumadinho/Galho do Miguel

Essa sequência corresponde às Formações SopaBrumadinho e Galho do Miguel, definidas por Pflug (1965, 1968). O contato basal dessa sequência é uma discordância. Ela aflora nas porções proximais da bacia, onde é caracterizada pela incisão de vales aluviais (Fig. 5). Essa discordância passa em direção ao centro da bacia para uma superfície transgressiva, aqui

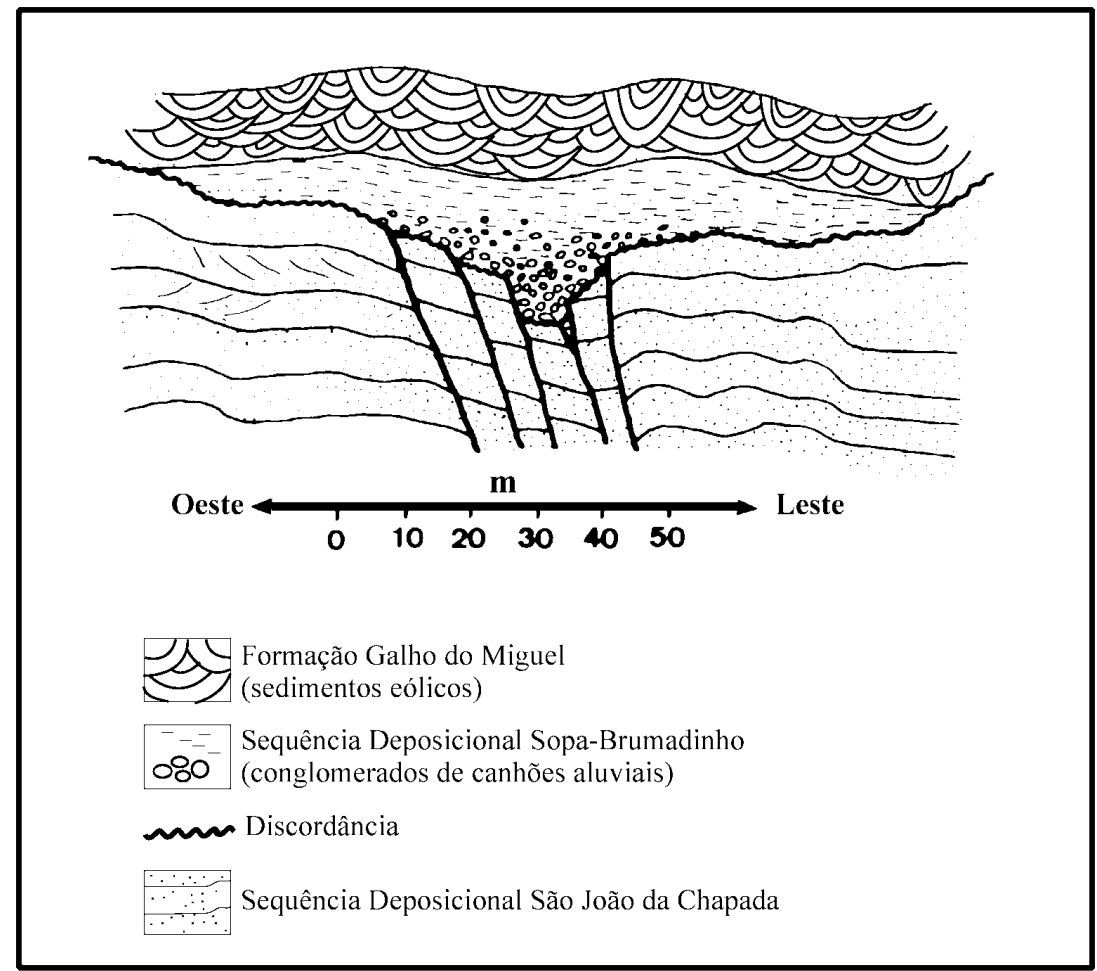

Figura 5: Perfil geológico da discordância basal da Sequência Deposicional Sopa-Brumadinho/Galho do Miguel.

Figure 5: Geologic cross-section of the basal unconformity of the Sopa-Brumadinho/Galho do Miguel Depositional Sequence. 
interpretada como uma conformidade correlativa (sensu Mitchum et al., 1977). Nessas porções a sedimentação é por acreção vertical de argila e silte muito fino. As intercalações de arenitos existentes dentro dos pelitos foram interpretadas como depósitos oriundos de correntes de turbidez em ambiente lacustrino (MartinsNeto, 1993).

Um sistema deltáico soterra progressivamente, para leste, os sedimentos pelíticos basais. As sucessões são granocrescentes, caracterizadas por arenitos na base e conglomerados com diamante detrítico no topo. Esses deltas foram alimentados pelos detritos oriundos dos vales entrincheirados nas cabeceiras aluviais. MartinsNeto (1993) descreveu um contato do tipo onlap para os arenitos que recobrem o sistema deltáico. Estes arenitos foram originados em plataformas marinhas influenciadas por correntes de marés (Fogaça \& Almeida-Abreu, 1982). Sedimentos eólicos recobrem concordantemente os sedimentos marinhos e marcam o fim da fase rift da Bacia Espinhaço e um período de estabilidade tectônica (Uhlein, 1991). Os arenitos eólicos correspondem a Formação Galho do Miguel de Pflug (1965, 1968).

\section{Sequência Deposicional Conselheiro Mata}

Esta sequência corresponde ao grupo homônimo definido por Dossin et al. (1984) e em conjunto corresponde às cinco formações de topo da coluna estabelecida por Pflug $(1965,1968)$. Essa sequência é transgressiva porque na base afloram lutitos e siltitos, depositados predominantemente por acreção vertical, recobrindo, conformavelmente, os campos de dunas eólicas da sequência inferior. O seu contato basal é uma superfície transgressiva, aqui interpretada como um limite de sequências. Não foram observados depósitos aluviais do tipo conglomerado basal. A interpretação de que a transgressão observada na base da sequência indica o ínício da fase flexural da evolução da Bacia Espinhaço foi inicialmente aventada por Chang et al. (1988), e posteriormente por Uhlein (1991), AlmeidaAbreu (1993), Schobbenhaus (1993) e Martins-Neto (1993).

Em um perfil geológico E-W, desde Diamantina até Conselheiro Mata, essa sequência apresenta o registro de três transgressões marinhas intercalados por períodos progradacionais (Dupont, 1995). A partir da base, recobrindo os metalutitos basais, sucedem-se sedimentos psamíticos, principalmente arcóseos, depositados em ambiente de praia, inter-maré e plataforma. Nova transgressão fica evidente na medida que esses psamitos apresentam gradual diminuição de granulometria em direção ao topo e transicionam para siltitos cinzas intercalados com lutitos de côr negra, principalmente depositados por processos de decantação e acreção vertical. Novo período progradacional é demostrado pela transição vertical granocrescente de lutitos distais para arenitos com marcas de onda e correntes intercalados com lutitos até arenitos finos a médios com estratificação cruzada acanalada. Segundo Dupont (1995) esses arenitos representam um sistema fluvial entrelaçado, entretanto não descartamos a hipótese de que esses depósitos registrem frentes de sistemas deltáicos. Lutitos com intercalações de siltitos recobrem, transicionalmente, esses arenitos. No topo da sequência, dolomitos estromatolíticos, em lentes ou na forma de bancos de espessura de poucos metros, foram observados por Schöll \& Fogaça (1979). Esses depósitos foram interpretados por Dupont (1996) como o registro de paleorecifes. Os dolomitos do topo da Bacia Espinhaço apresentam-se carstificados e suas cavidades foram preenchidas por arenitos com seixos da Bacia São Francisco.

\section{A Bacia São Francisco}

Os sedimentos Neoproterozóicos da Bacia São Francisco afloram em extensas áreas no norte do estado de Minas Gerais. Essa bacia é limitada, na base, por uma discordância com os sedimentos da Bacia Espinhaço (Pflug, 1965; 1968; Pflug \& Renger, 1973). Para leste, os sedimentos dessa bacia repousam discordantemente sobre o complexo de rochas do embasamento. Litoestratigraficamente, as rochas da Bacia São Francisco correspondem em conjunto aos grupos Macaúbas e Bambuí, Formação Salinas e a todas unidades que foram depositadas recobrindo a discordância basal e/ou conformidade correlativa dessa bacia.

A idade do início da deposição na Bacia São Francisco é balizada por diques de rochas básicas que cortam os sedimentos da Bacia Espinhaço, mas não cortam os sedimentos sobrepostos. Datações U/Pb em zircões dessas rochas forneceram a idade de $0.9 \mathrm{Ga}$ (Machado et al., 1989). Essa idade é interpretada como a idade mínima para a deposição dos sedimentos da Bacia Espinhaço e o começo da evolução da Bacia São Francisco.

O desenvolvimento da discordância basal dessa bacia está relacionado à instalação de um ambiente glacial no Craton do São Francisco. Vestígios dessa glaciação foram reportados nos sedimentos do Grupo Macaúbas por Isotta et al. (1969), Walde (1976), Hettich (1977), Karfunkel \& e Karfunkel (1977), Karfunkel \& Hoppe (1988), entre outros. Dessa forma, com a glaciação, grandes massas de águas, oriundas dos oceanos, foram tranferidas para os continentes, gerando eustasia negativa com consequente rebaixamento relativo do nível do mar, exposição subaérea de sedimentos litificados da Bacia Espinhaço, erosão dos mesmos e o desenvolvimento da discordância basal.

De oeste para leste, recobrindo a discordância basal da Bacia São Francisco, a deposição dos sedimentos ocorreu nos seguintes paleoambientes: 1. zonas proximais das geleiras, em contato com os sedimentos previamente litificados da Bacia Espinhaço, bem como do embasamento (Karfunkel \& Hoppe, 1988). Pavimentos estriados nas rochas sedimentares de topo da Bacia Espinhaço foram descritos por Walde (1976) 
na borda leste da Serra do Cabral e apontam a direção de transporte das geleiras de oeste para leste. Recobrindo os pavimentos glaciais ocorrem tilitos que apresentam seixos angulosos de arenitos, rochas carbonáticas (Espinhaço ?), gnaisses, granitos e quartzo de veio. A composição dos grãos detríticos da matriz dos tilitos apresenta grande quantidade de fragmentos líticos estáveis de rochas plutônicas, bem como grãos detríticos de feldspatos e quartzo (Karfunkel \& Hoppe, 1988); 2. área continental, com deposição de tilitos e desenvolvimento de planícies de out-wash; 3. zona transicional entre o continente e o mar, com aporte de detritos oriundos das geleiras (Karfunkel \& Hoppe, 1988); 4. para leste ocorre uma zona distal, na qual os sedimentos dessa bacia recobrem o complexo de rochas ígneas-migmatíticas do embasamento. A deposição de turbiditos em águas profundas caracteriza esse ambiente distal (Hettich, 1977; Karfunkel \& Karfunkel, 1977; Pedrosa-Soares et al., 1992a; 1992b). Nessas porções o limite de sequências é representado pela conformidade correlativa à discordância.

O mapeamento geológico em escala 1:25.000 e o levantamento de colunas estratigráficas detalhadas, conduzidos nas adjacências de Conselheiro Mata, permitiram elaborar a reconstrução paleogeográfica da discordância da base da Bacia São Francisco. Essa reconstrução paleogeográfica, apresentada na Figura 6, localiza-se na área 2 - zona continental com deposição de tilitos e planícies de out-wash. Nessa área, os dolomitos do topo da Bacia Espinhaço apresentam-se carstificados. As cavidades do paleocarste foram preenchidas por areias e seixos. Recobrindo a discordância e os dolomitos carstificados ocorre uma sucessão arenosa e conglomerática com estratificação interna plano-paralela bem desenvolvida, interpretada como uma planície de out-wash.

A água do escoamento superficial, oriunda do degelo sazonal das geleiras continentais, percolou o embasamento dolomítico do topo da Bacia Espinhaço, carstificando-o. Ao percolá-lo, a carga trativa da corrente fluvial foi depositada em superfície ou penetrou o paleocarste, ficando ali aprisionada. Pode-se concluir que os processos de carstificação foram provocados por um rebaixamento relativo do nível do mar, que causou a exposição subaérea dos sedimentos químicos do topo da Bacia Espinhaço.

Devido a deglaciação ocorreu uma mudança eustática positiva que foi a responsável pela transgressão marinha sobre o Craton do São Francisco. Extensas plataformas carbonáticas e sedimentação de finos por

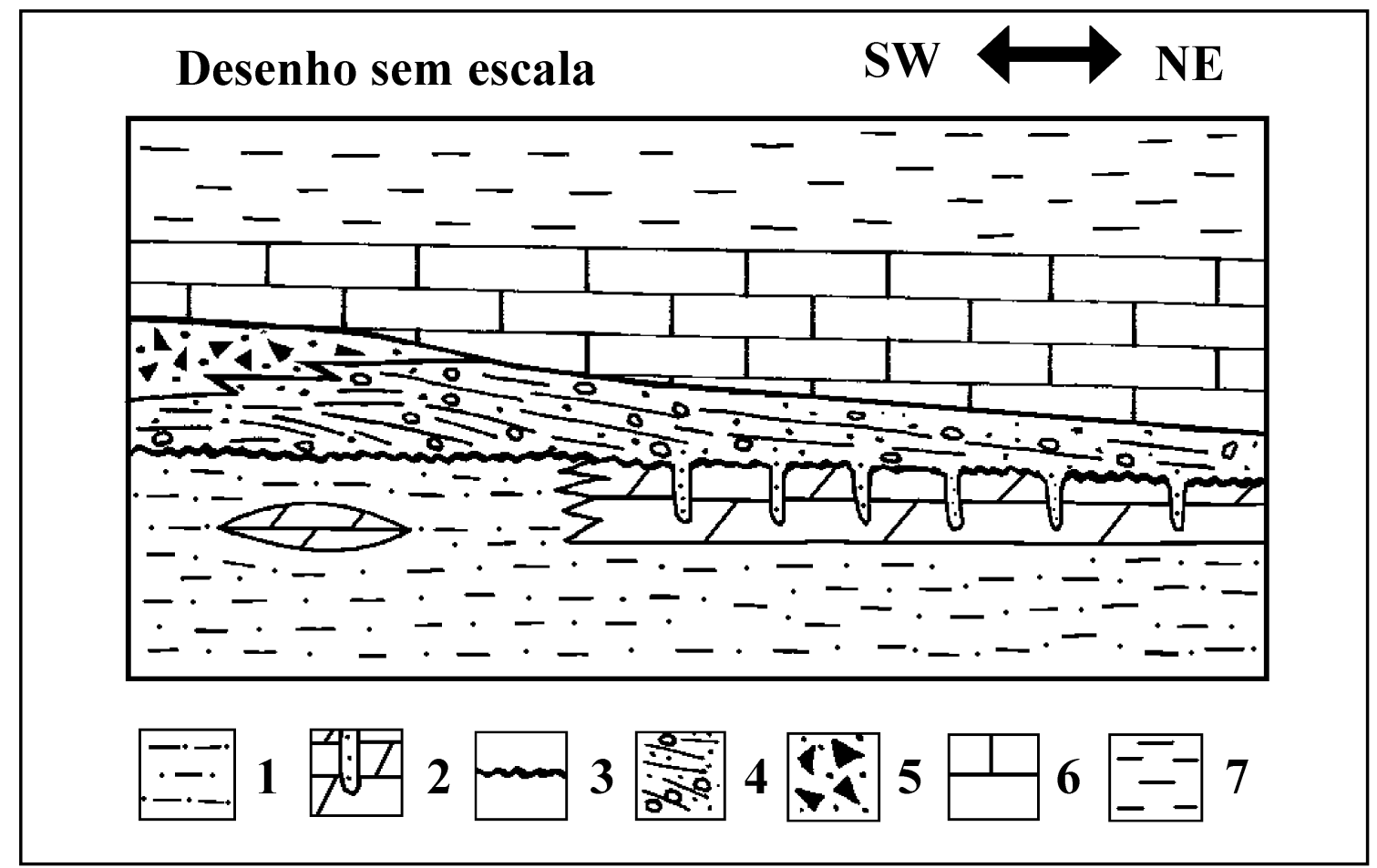

Figura 6: Perfil geológico esquemático da discordância basal da Bacia São Francisco na região de Conselheiro Mata. Legenda: 1=lutitos do topo da Bacia Espinhaço, 2=dolomitos carstificados, 3=discordância, 4=sistema fluvial entrelaçado (out-wash plain), 5=diamictitos glaciais, $6=$ rochas carbonáticas e 7=lutitos da Bacia São

Francisco.

Figure 6: Schemmatic cross-section of the basal unconformity of the São Francisco Basin in the Conselheiro Mata region. Key: $1=$ lutites of the uppermost portions of the Espinhaço Basin, $2=$ dolomites with paleokarst, $3=$ unconformity, 4=fluvial braided system (out-wash plain), 5=Glacial diamictites, $6=$ carbonatic rocks and $7=$ 
acreção vertical caracterizam a deposição durante essa etapa evolutiva. Os sedimentos da Bacia São Francisco são separados das coberturas fanerozóicas por uma discordância desenvolvida durante o soerguimento da Cordilheira do Espinhaço, que ocorreu devido aos efeitos compressivos da Orogenia Brasiliana.

\section{PROVENIÊNCIA DOS SEDIMENTOS PROTEROZÓICOS}

A proveniência dos sedimentos da Bacia Espinhaço é indicativa da existência de uma área fonte intrabasinal e outra extrabasinal. A área fonte intrabasinal corresponde aos sedimentos da própria bacia, previamente litificados e retrabalhados localmente. A área fonte extrabasinal corresponde ao embasamento, ou seja, rochas graníticas associadas com rochas vulcânicas, BIFs e sedimentos químicos, representativos de uma sequência greenstone (Silva, 1995a). A análise modal dos arcóseos das sequências deposicionais Basal, Olaria e Natureza são indicativos de que o embasamento granítico foi intemperizado quimicamente na área fonte, com destruição seletiva de plagioclásio e fornecimento de quartzo e K-feldspato em abundância. Conforme os diagramas de Dickinson (1970, 1985), a área fonte estava posicionada em um bloco continental, localizado dentro do Craton do São Francisco, a oeste da Bacia Espinhaço.

Os sedimentos basais da Bacia São Francisco provêm em grande parte do retrabalhamento pelas geleiras dos depósitos previamente litificados da Bacia Espinhaço. Entretanto, a existência de seixos de granitos e gnaisses, presentes nos tilitos, indica que o nível de erosão nos vales glaciais foi profundo o suficiente para exumar por completo, pelo menos localmente, os sedimentos sotopostos da Bacia Espinhaço e retrabalhar o embasamento cratônico.

\section{CONCLUSÕES}

A proposta aloestratigráfica, apresentada na Figura 1, baseia-se no fato de que as discordâncias representam verdadeiros limites de sequências. Entende-se que o retrabalhamento local de sedimentos previamente litificados é um argumento indiscutível a favor da existência de sucessivos ciclos de deposição e litificação intercalados por períodos erosivos. A proposta de subdivisão dos registros da cobertura do Craton do São Francisco em sequências deposicionais permitiu individualizar duas bacias Proterozóicas distintas: Bacia Espinhaço e Bacia São Francisco (Fig. 7).

A Bacia Espinhaço é interpretada como uma bacia

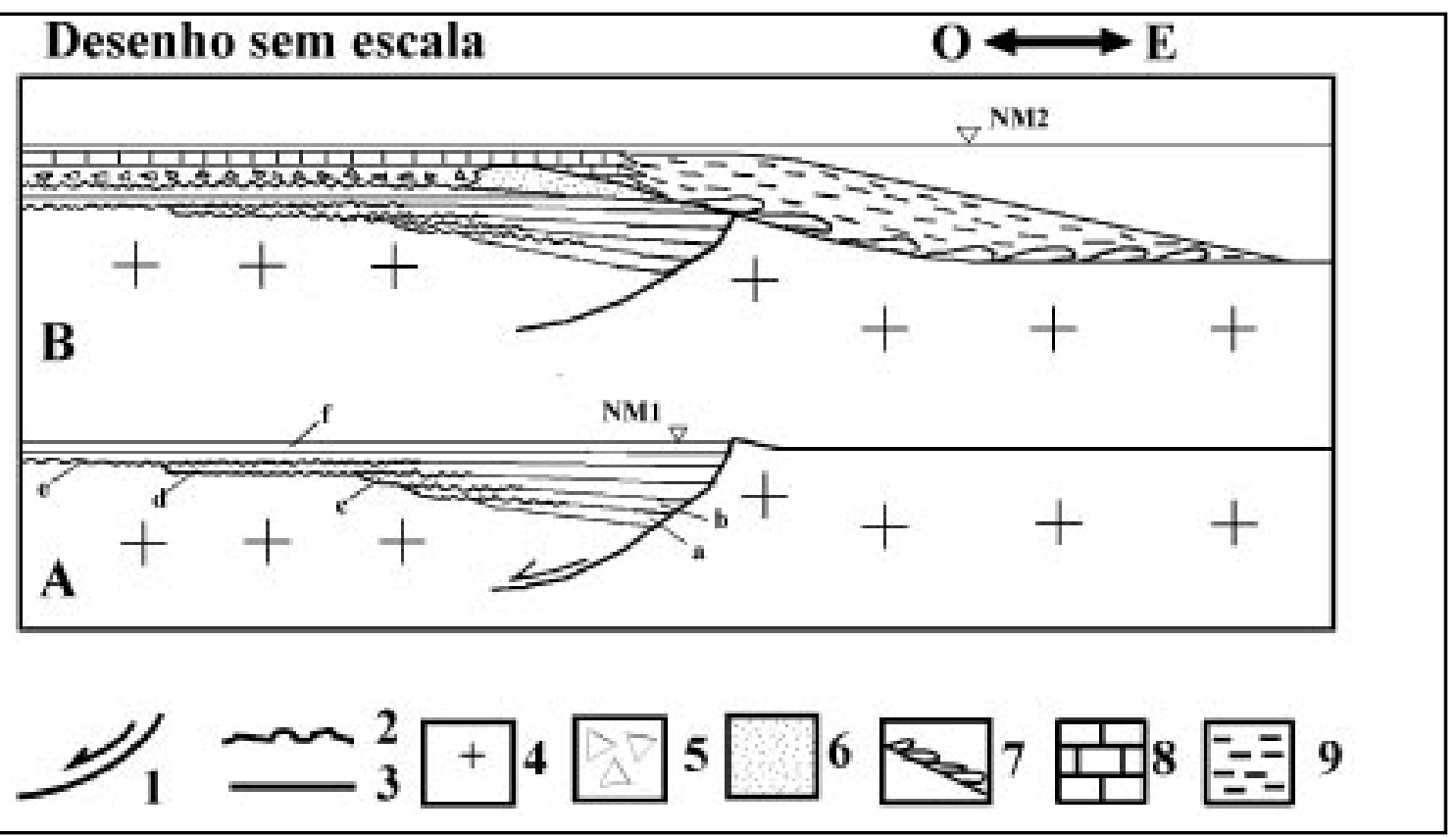

Figura 7: Evolução tectônica das bacias Espinhaço e São Francisco em Minas Gerais. (A) Bacia Espinhaço. Sequências deposicionais: $a=$ Basal, $b=$ Olaria, $c=$ Natureza, $d=$ São João da Chapada, e=Sopa-Brumadinho/ Galho do Miguel e $f=$ Conselheiro Mata. NM1=nível do mar no final da evolução da Bacia Espinhaço. (B) Bacia São Francisco. NM2=nível do mar no final da evolução da Bacia São Francisco. Legenda: 1=falha lístrica basal, 2=discordância, 3=conformidade correlativa, 4=embasamento (pré-Transamazonico), $5=$ depósitos glaciais, $6=$ sistema fluvial entrelaçado (out-wash plain), 7=leques turbidíticos distais, 8=rochas carbonáticas e 9=lutitos da Bacia São Francisco.

Figure 7: Tectonic evolution of the Espinhaço and São Francisco basins in Minas Gerais. (A) Espinhaço Basin. Depositional sequences: $a=$ Basal, $b=$ Olaria, $c=$ Naturez $a, d=$ São João da Chapada, $e=$ Sopa-Brumadinho/ Galho do Miguel and $f=$ Conselheiro Mata. NMI=sea level at the end of the evolution of the Espinhaço Basin. (B) São Francisco Basin. NM2 = sea level at the end of the evolution of the São Francisco Basin. Key: 1=basal listric fault, $2=$ unconformity, $3=$ correlative conformity, 4=basement (pré-Transamazonic), 5=glacial deposits, $6=$ fluvial braided system (out-wash plain), 7=distal turbiditic fans, $8=$ carbonatic rocks and $9=$ lutites of the São 
intracratônica do tipo rift. Sua evolução abrange o período temporal entre $1.8 \mathrm{Ga}$ e, aproximadamente, 0.9 Ga. Através do estudo detalhado dos conglomerados basais concluiu-se que: 1. as discordâncias foram desenvolvidas durante períodos tafrogênicos e representam superfícies de erosão subaérea; 2. os conglomerados basais representam depósitos sintectônicos, controlados pelos efeitos conjugados do soerguimento da área fonte e da subsidência do depocentro. Esse tipo de comportamento corresponde ao de uma rampa inclinada de uma falha lístrica basal. Cada movimento ao longo da falha provocou a rotação da rampa e em consequência os canais aluviais entrincheiraram-se nas cabeceiras e alimentaram os leques aluviais progradacionais. No caso específico da Sequência Deposicional Sopa-Brumadinho/Galho do Miguel, o entrincheiramento dos canais aluviais alimentou diretamente os leques deltáicos ricos em diamantes detríticos.

Durante a fase pós-rift foram depositados os sedimentos transgressivos da Sequência Deposicional Conselheiro Mata. Essa sequência não apresenta conglomerados na base e seu desenvolvimento está relacionado a movimentos de subsidência flexural, que induziu uma transgressão e a consequente deposição de sedimentos marinhos-rasos diretamente acima de sedimentos continentais. Estudos de paleocorrentes são indicativos de que, inicialmente, o nível do mar se encontrava a S-SE da Bacia Espinhaço e que inundou o rift, e durante a fase flexural, fase pós-rift, transgrediu sobre o Craton do São Francisco. Provavelmente, a Bacia Espinhaço adquiriu uma morfologia de um golfo marinho, limitado a leste pela falha lístrica basal e a oeste pela rampa tectônica.

A Bacia São Francisco está separada da Bacia Espinhaço e seu embasamento por uma discordância de carater interregional. Sua evolução abrange o período compreendido entre 0.9 e $0.6 \mathrm{Ga}$. Durante esse intervalo a bacia adquiriu uma morfologia de margem passiva, com desenvolvimento de plataforma, talude e regiões pelágicas distais. $\mathrm{O}$ desenvolvimento da discordância da base da bacia está relacionado a instalação de uma glaciação sobre o Craton do São Francisco em Minas Gerais. Com a glaciação ocorreu uma mudança eustática negativa que induziu o rebaixamento relativo do nível do mar. O transporte de detritos ocorreu pela ação de geleiras continentais e ambientes periféricos que alimentavam as correntes de turbidez em porções distais da bacia. Devido a posterior deglaciação, movimentos de eustasia positiva causaram uma subida relativa do nível do mar sobre o Craton do São Francisco. Sedimentação carbonática plataformal e deposição de finos por acreção vertical caracterizam essa transgressão.

Similar arcabouço estratigráfico e tectônico apresentado pelas Bacias Espinhaço e São Francisco em Minas Gerais foram também descritos para as bacias homônimas no Estado da Bahia (Silva, 1996) e para as
Bacias São João del Rei e Andrelândia no sul de Minas Gerais (Ribeiro \& Silva, 1996). De uma forma geral, foi constatado uma correspondência impressionante e um sincronismo entre a deposição das sequências descritas aqui com aquelas que constituem estas bacias. Esse fantástico quadro cronoestratigráfico nos remete à evolução das bacias intracratônicas de Idade Fanerozóica e um paralelo comparativo deve ser traçado. Entende-se que o conjunto de dados levantados até o presente momento a respeito da evolução sedimentar durante o Proterozóico ainda é fragmentário. Entretanto, o uso de conceitos e métodos de Estratigrafia de Sequências pode mudar esse quadro e iluminar a história geológica das coberturas do Craton do São Francisco.

\section{Agradecimentos}

Quero expressar minha gratidão aos inúmeros alunos de geologia de diversas escolas do Brasil, que me acompanharam e incentivaram durante os últimos 11 anos de trabalhos de campo. Gostaria também de agradecer aos Profs. L.G. Knauer e F.E. Renger do CPMTC/IGC/UFMG, ao prof. B.B. Brito Neves do IG/ USP e ao Prof. A. Ribeiro do IGEO/CCMN/UFRJ pela cuidadosa revisão crítica do texto.

\section{REFERÊNCIAS BIBLIOGRÁFICAS}

ALMEIDA-ABREU, P.A. 1989. Geologia das quadrículas de Onça e Guinda (Gouveia, M.G.) região meridiana central da Serra do Espinhaço Meridional. M.Sc. Thesis, Univ. Fed. Rio de Janeiro, 75 pp. (não publicado).

ALMEIDA-ABREU, P.A. 1993. A evolução geodinâmica da Serra do Espinhaço Meridional, Minas Gerais, Brasil. Ph.D. Thesis, Univ. Freiburg, Freiburg, Alemanha, 150 pp. (não publicado).

BRITO-NEVES, B.B.; KAWASHITA, K.; CORDANI, U.G. \& DELHAL, J. 1979. A evolução geocronológica da Cordilheira do Espinhaço, dados novos e integração. Rev. Bras. Geoc., 9: 71-85.

BRITO-NEVES, B.B.; SÁ, J.M.; NILSON, A.A. \& BOTELHO N.F. 1995 A Tafrogênese Estateriana nos blocos paleoproterozóicos da América do Sul e processos subsequentes. Geonomos, Vol. 3(2): 1-21.

CHANG, H.K.; MIRANDA, F.P.; MAGALHÃES, L. \& ALKMIN, F.F. 1988. Considerações sobre a evolução da Bacia do São Francisco. Anais do $35^{\circ}$ Cong. Bras. Geol., Vol. 5: 2076-2090.

COMISSÃO NORTE-AMERICANA DE NOMENCLATURA ESTRATIGRÁFICA. 1983. North American Stratigraphic code. Bull. Geol. Soc. Am., 67: 841-875.

DICKINSON, W.R. .1970. Interpreting detrital modes of graywacke and arkose. Jour. Sediment. Petrol., 63: 2164-2182.

DICKINSON, W.R. 1985. Interpreting provenance relations from detrital modes of sandstones. In: G.G.D. ZUFFA (Editor), Provenance of Arenites, Reidel: 333-361.

DOMINGUEZ, J.M.L. 1993. As coberturas do Craton do São Francisco: uma abordagem do ponto de vista de análise de bacias. In: J.M.L. Dominguez e A. Mise (Editores), $2^{0}$ Simp. Craton do São Francisco, Soc. Bras. Geol.: 137-159.

DOSSIN, I.A. \& DOSSIN T.M. 1995. Supergrupo Espinhaço: modelo de evolução geodinâmica, Geonomos, 3(1): 19-26.

DOSSIN, I.A.; UHLEIN, A. \& DOSSIN, T.M. 1984. Geologia da Faixa móvel Espinhaço em sua porção meridional, M.G. Anais do $33^{0}$ Cong. Bras. Geol., Vol. 7: 3118-3132.

DOSSIN, I.A.; DOSSIN, T.M.; CHARVET, J.; COCHERIE A. \& ROSSI, P. 1993. Single-zircon dating by step-wise Pb- 
evaporation of Middle Proterozoic magmatism in the Espinhaço Range, southeastern São Francisco Craton (Minas Gerais, Brazil). Anais $2^{0}$ Simp. do Craton do São Francisco: 39-42.

DOSSIN, T.M. 1994. Associations volcano-plutoniques de L'Espinhaço meridional (SE-Brésil): un exemple d'evolution de la crôute Protérozoic. Ph.D. Thesis, Univ. Orléans, França, 177 pp (não publicado).

DUPONT, H. 1995. O Grupo Conselheiro Mata no seu quadro Paleogeográfico e estratigráfico. Anais do $8^{0}$ Simp. Geol. Minas Gerais: 9-10

DUPONT, H. 1996. O Supergrupo São Francisco entre a Serra do Cabral e as Serras do Espinhaço e de Minas, estudo estratigráfico e estrutural e relações de contato com o Supergrupo Espinhaço. Anais do $39^{\circ}$ Cong. Bras. Geol., Vol. 5: 489-493.

FOGAÇA, A.C.C. \& ALMEIDA-ABREU, P.A. 1982. Depósitos de planícies de marés na Formação Sopa-Brumadinho, Cordilheira do Espinhaço, Minas Gerais, Brasil. Anais do $5^{0}$ Cong. LatinoAmericano Geol., Vol. 2: 373-388.

FOGAÇA, A.C.C.; ALMEIDA-ABREU, P.A. \& SCHORSCHER, H.D. 1984. Estratigrafia da sequência supracrustal arqueana na porção mediana central da Serra do Espinhaço, M.G. Anais do $33^{0}$ Cong. Bras. Geol., Vol. 2: 2652-2667.

HETTICH, M. 1977. A glaciação Proterozóica no centro-Norte de Minas Gerais. Rev. Bras. Geoc., 7: 87-101.

HOFFMANN, C. 1983. The Archean peraluminous Gouveia Granite: its structure, geochemistry and phase petrology (Serra do Espinhaço, Minas Gerais, Brazil). N. Jb. Min., 146(2): 359-371.

HOPPE, A. 1978. Beiträge zur geologie und Paläogeographie der südlichen Serra do espinhaço (Minas Gerais, Brasilien). Ph.D. Thesis, Univ. Fraiburg, Alemanha, 102 pp (não publicado).

HOPPE, A.; LAMMERER, R.B.; MÜLLER, G. PFLUG, R. \& DIEFENBACH, K.W. 1987. Aspectos da geologia e petrologia da Serra do Espinhaço-Sul e entre o Quadrilátero Ferrífero e o Cinturão Móvel Brasiliano no Sul do Espírito Santo, Brasil. In: H. Miller (Editor), Investigaciones Alemanes Recientes em Latinoamérica. Proyectos de la Deutsche Forchungsgemeinschaft, DFG/Boldt, Boppard, 8597.

ISOTTA, C.A.L.; ROCHA-CAMPOS, A.C. \& YOSHIDA, R. 1969. Striated pavement of the upper Precambrian glaciation in Brazil. Nature, 222: 466-468.

KARFUNKEL, J. \& HOPPE, A. 1988. Late Proterozoic glaciation in Central-Eastern Brazil: synthesis and model. Paleogeography, Paleoclimatology, Paleoecology, 65: 1-21.

KARFUNKEL, B \& KARFUNKEL, J. 1977. Faziell Entwicklung der mittleren Espinhaço-zone mit besondere Berücksichitigung des Tillit-Problems (Minas Gerais, Brasilien). Geol. Jahrb., $\mathrm{B}(24)$ : 3-91.

KRUMBEIN, W.C. \& SLOSS, L.L. 1956. Stratigraphy and sedimentation. W.H. Freeman and Co., San Francisco, 497 pp.

KNAUER, L.G. \& SCHRANK, A. 1993. A origem dos filitos hematíticos da Serra do Espinhaço Meridional, Minas Gerais. Geonomos, 1: 33-38.

MACHADO, N.; SCHRANK, A.; ABREU, F.R.; KNAUER, L.G. \& ALMEIDA-ABREU, P.A. 1989. Resultados preliminares da geocronologia U/Pb na Serra do Espinhaço Meridional. Bol. Soc. Bras. Geol., Minas Gerais, 10: 171-174.

MARTINS-NETO, M.A. 1989. Sistemas deposicionais e paleogeografia das formações basais do Supergrupo Espinhaço (São João da Chapada e Sopa-Brumadinho) na região de Diamantina/Costa Sena, Minas Gerais, Brazil. M.Sc. Thesis, Univ. Fed. da Bahia, 88 pp. (não publicado).

MARTINS-NETO, M.A. 1993. The sedimentary evolution of a Proterozoic rift basin: the basal Espinhaço Supergroup, southern Serra do Espinhaço, Minas Gerais, Brazil. In: R. Pflug e H. Ulmer (Editores), Freiburger Geowissenchaftliche Beiträge, 4, $155 \mathrm{pp}$.

MARTINS-NETO, M.A. 1994. Braidplain sedimentation in a Proterozoic rift basin: the São João da Chapada Formation, southeastern Brazil. Sediment. Geol., 89: 1222-1243.

MITCHUM, R.M.JR.; VAIL, P.R. \& THOMPSON, S. 1977. Seismic stratigraphy and global changes of sea level, part two: the deposicional sequence as a basic unit for stratigraphic analysis. In: C.E. Payton (Editor), Seismic Stratigraphy-Applications to Hydrocarbon Exploration. Mem. Am. Assoc. Petrol. Geol., 26: 53-62.

PEDROSA-SOARES A.C.; NOCE, C.M.; VIDAL, P. \& MONTEIRO, R.B.P. 1992a. Discussão sobre novo modelo tectônico para a Faixa Aracuaí-Oeste Congolesa. Rev. Esc. Minas, 45: 38-40.

PEDROSA-SOARES, A.C.; NOCE, C.M.; VIDAL, P.; MONTEIRO, R.B.P. \& LEONARDOS, O.H. 1992b. Toward a new tectonic model for the Late Proterozoic Araçuaí (SE Brazil)-West Congolian (SW Africa) Belt. Journal of South American Earth Sciences, 2(1/2): 33-47.

PFLUG, R. 1965. A geologia da parte meridional da Serra do Espinhaço e zonas adjacentes. Bol. Dep. Nac. Prod. Min., Div. Geol. Min., 226, 51 pp.

PFLUG, R. 1968. Observações sobre a estratigrafia da Série Minas na região de Diamantina, Minas Gerais. Dep. Nac. Prod. Min., Div. Geol. Min., 142, 20 pp.

PFLUG, R. \& RENGER, F.E. 1973. Estratigrafia e evolução geológica da Margem SE do Craton Sanfranciscano. Anais do $27^{0}$ Cong. Bras. Geol., Vol. 2: 5-19.

RENGER, F.E. 1970. Fazies und Magmatismus der Minas-Serie in der südlichen Serra do Espinhaço, Minas Gerais, Brasilien, Geol. Rundsch., 59: 1253-1292.

RIBEIRO, A. \& SILVA, R.R. 1996. Possíveis correlações entre bacias Proterozóicas a sul e sudeste do Craton do São Francisco, Minas Gerais. Anais do $39^{\circ}$ Cong. Bras. Geol., Vol. 6: 11-14.

SCHOBBENHAUS, C. 1993. O Proterozóico Médio no Brasil com ênfase à região Centro-Leste: uma revisão. Ph.D. Thesis, Univ. Freiburg, Freiburg, Alemanha, 166 pp. (não publicado).

SCHÖLL, W.Ü. \& FOGAÇA, A.C.C. 1981. Mapa geológico da Quadrícula de Guinda, escala 1:25.000. Proj. Map. Geol. Espinhaço Meridional, Dep. Nac. Prod. Min./Centro Geol. Eschwege, 44 pp. (não publicado).

SCHÖLL, W.Ü. 1980. Estratigrafia, sedimentologia e paleogeografia na região de Diamantina (Serra do Espinhaço, Minas Gerais, Brasil). Forsch. Geol. Paläont., 51: 223-240.

SCHÖLL, W.Ü. \& FOGAÇA, A.C.C. 1979. Estratigrafia da Serra do Espinhaço na região de Diamantina. Bol. Soc. Bras. Geol., 1: 55-71.

SILVA, R.R. 1993. Seqüências estratigráficas da fase rift do Supergrupo Espinhaço, Diamantina, Minas Gerais. Anais do $2^{0}$ Simp. do Craton do São Francisco: 154-157.

SILVA, R.R. 1994. Deposicional sequences of the rift phase of the Proterozoic Espinhaço Supergroup, in the area between Diamantina and Gouveia, Minas Gerais, Brazil. $14^{\circ}$ Inter. Sedim. Cong., G: 75-76.

SILVA, R.R. 1995a. Contribution to the stratigraphy and paleogeography of the lower Espinhaço Supergroup (Mesoproterozoic) between Diamantina and Gouveia, Minas Gerais State, Brazil. In: R. Pflug e H. Ulmer (Editores), Freiburger Geowissenchaftliche Beiträge, 8, $115 \mathrm{pp}$.

SILVA, R.R. 1995b. The lower Espinhaço Supergroup in the area between Diamantina and Gouveia, Minas Gerais, Brazil: an example of intracratonic rift basin. Anais do $8^{\circ}$ Simp. Geol. Minas Gerais: 124-126.

SILVA, R.R. 1996. Correlation of the depositional sequences of the Proterozoic Espinhaço and São Francisco Basin System, in Minas Gerais and Bahia States. Anais do $39^{\circ}$ Cong. Bras. Geol., Vol. 6: 142-144.

SLOSS, L.L. 1963. Sequences in the cratonic interior of North America. Bull. Geol. Soc. Am., 74: 94-114.

SLOSS, L.L.; KRUMBEIN, W.C. \& DAPPLES, E.C. 1949. Integrated facies analysis. Mem. Geol. Soc. Am., 39: 91-123.

SUBCOMITE INTERNACIONAL DE CLASSIFICAÇÃO ESTRATIGRÁFICA (SALVADOR, A., CHAIRMAN). 1987. Unconformity-bounded stratigraphic units. Bull. Geol. Soc. Am., 98: 232-237.

TROMPETTE, R; UHLEIN, A.; SILVA, M.E. \& KARMANN, I. 1992. The Brasiliano São Francisco Craton revisited (central Brazil). Journal of South-American Earth Sciences: 6: 49-57.

UHLEIN, A. 1991. Transição craton-faixa dobrada: exemplo do Craton do São Francisco e da Faixa Araçuaí (Ciclo Brasiliano) no Estado de Minas Gerais. Aspectos estratigráficos e estruturais. Ph.D. Thesis, Univ. São Paulo, 295 pp. (não publicado).

UHLEIN, A.; TROMPETTE, R. \& SILVA, M.E. 1986. Estruturação tectônica do Supergrupo Espinhaço na região de Diamantina (M.G.). Rev. Bras. Geoc., 16: 212-216.

VAIL, P.R.; MITCHUM, R.M.JR. \&THOMPSON, S. 1977. Seismic stratigraphy and global changes of sea-level, part three: relatives changes of sea level from coastal onlap. In: C.E. Payton (Editor), Seismic Stratigraphy-Applications to Hydrocarbon Exploration. Mem. Am. Assoc. Petrol. Geol., 26: 63-82.

WALDE, D. 1976. Neue Hinweise für eine proterozoisch Vereisung in Ostbrasilien. Münster Forsch. Geol. Paläont. H.(38/39): 47-59. 